\title{
Intérêt de l'indice de végétation des satellites NOAA-AVHRR pour le suivi des cultures (résultats d'une étude dans la basse vallée du Rhône)
}

Jean-Pierre LAGOUARDE, Bernard SEGUIN, Sylvie CLINET*, Soledad GANDIA** \& Yann KERR***

I.N.R.A., Station de Bioclimatologie, Centre de Recherches d'Avignon, B.P. 91, F. 84140 Montfavet

(*) Institut de Géographie d'Aix, Université Aix-Marseille II, 29, avenue R. Schumann, F. 13621 Aix-enProvence

(**) Departamento de Termologia, Facultad Fisicas, Universitad de Valencia, Burjasot (Valencia), Espagne (***) LERTS, 18, avenue Edouard Belin, F. 31055 Toulouse Cedex

Par rapport aux satellites d'observation de la Terre (Landsat, SPOT) à forte résolution spatiale, mais faible répétitivité temporelle, les satellites météorologiques NOAA-AVHRR ont l'avantage de permettre, grâce à 4 passages par jour, un suivi des cultures au cours de la saison de végétation. L'inconvénient majeur est la résolution spatiale limitée à $1 \mathrm{~km}^{2}$. Une étude a été effectuée sur les années 1983 et 1984 pour évaluer l'intérêt des indices de végétation dérivés des données NOAA, parallèlement aux données d'IR thermique analysées par ailleurs. Localisée dans la basse vallée du Rhône, elle a permis de caractériser l'évolution différenciée de petites régions au mode d'occupation du sol nettement contrasté. Une étude plus fine montre la possibilité de suivre les cycles culturaux dominants sur les surfaces quasiment homogènes et d'envisager une quantification de la production de matière sèche par les relations existant entre indice de végétation et productivité.

Mots clés additionnels : Télédétection, couvert végétal. satellites for monitoring crop growth.

Compared to earth observation satellites (Landsat, SPOT) with high ground resolution but low temporal frequency, NOAA-AVHRR meteorological satellites allow crop conditions to be monitored through the growing season thanks to 4 passes per day. The major limitation lies in the spatial resolution, limited to $1 \mathrm{~km}^{2}$. This study has been undertaken in order to test the value of NOAA-derived vegetation indices, in parallel to thermal IR data analysed elsewhere, for 1983 and 1984. Observations on the lower Rhone valley allowed characterization of the differential evolution of small regions with various land uses. A more detailed study at the smallest possible spatial scale opened the possibility of monitoring the growth cycles of the predominant crops within virtually homogeneous areas and of quantifying biomass production using relationship between vegetation indices and productivity.

Additional key words : Remote sensing, crop canopy.

\section{INTRODUCTION}

Dans le domaine de la télédétection satellitaire, l'intérêt des agronomes s'est surtout porté en France, jusqu'à présent, sur les satellites d'observation de la Terre (la série des Landsat essentiellement, puis SPOT) seuls susceptibles d'atteindre les résolutions spatiales (de l'ordre de $100 \mathrm{~m}$, puis $20 \mathrm{~m}$ ) compatibles avec le parcellaire à étudier. Cependant, la faible réso- lution temporelle de Landsat (18 à 16 jours) ou de SPOT (26 jours, mais avec possibilité de dépointage permettant, en théorie, des observations tous les 2 à 3 jours sur une zone sélectionnée) conduit essentiellement à des applications d'identification des cultures et de cartographie (SAINT et al., 1984) utilisant 3 ou 4 scènes au cours de la saison de végétation pour effectuer des classifications multitemporelles.

Par contre, les satellites météorologiques, soit géo- 
stationnaires (Meteosat), soit à défilement (série des NOAA), qui permettent d'obtenir des informations à forte résolution temporelle $(1 / 2 \mathrm{~h}$ dans le premier cas, 4 par jour pour le deuxième), présentent l'inconvénient d'une résolution spatiale limitée $(5 \mathrm{~km}$ et $1 \mathrm{~km})$, les réduisant essentiellement à des utilisations agroclimatologiques (SEGuIN, 1983). Dans le cas de NOAA cependant, des travaux récents ont mis en évidence l'intérêt des indices de végétation pour le suivi de la végétation à grande échelle sur l'ensemble du globe (parmi les nombreux travaux publiés, voir par exemple JUSTICE et al., 1985).

Il nous a donc paru intéressant d'évaluer l'apport de ce paramètre dans le Sud-Est de la France en complément des données de l'IR thermique qui ont fait l'objet d'un travail parallèle publié par ailleurs (VIDAL et al., 1986). Mentionnons qu'une étude a également été effectuée, dans ce même projet, sur la signification des indices de végétation NOAA dans le Sud-Ouest de la France (ANGLADE, 1985), et a mis en évidence leur intérêt pour la classification des petites régions agricoles par le biais de leurs systèmes culturaux.

\section{LES DONNÉES DISPONIBLES}

Les données disponibles sont acquises par les satellites NOAA-AVHRR (ce dernier sigle caractérisant le radiomètre Advanced Very High Resolution Radiometer, à bord de TIROS-N, puis des NOAA 6 à 9 à partir de 1978). Ces satellites à défilement, opérant par paires, permettent 4 passages par jour, 2 de nuit et 2 de jour (vers 8 h 30 et 14 h 30 TU). Pour cette étude, portant sur le Sud-Est de la France, au cours des années 1983 et 1984, seules ont été acquises des données du milieu de journée. Au cours de la première année (1983), 7 dates ont été obtenues pour la période juillet-septembre à titre d'essai. L'intérêt des données traitées nous a conduit à rechercher une couverture temporelle plus élevée en 1984: pour cette année, 2 scènes ont été acquises en janvier, puis 31 entre les mois d'avril et octobre, ce qui correspond approximativement au rythme souhaité de 2 acquisitions par semaine, à l'exception du mois de mai particulièrement nuageux cette année-là.

Les données, acquises au Centre de Météorologie Spatiale de Lannion, sont prétraitées au LERTS Toulouse : rectification géométrique, correction pour l'élévation solaire, conversion des images en luminances pour les courtes longueurs d'onde ; de plus, la température de surface est calculée comme étant une combinaison linéaire des températures radiatives $T_{4}$ et $T_{5}$ mesurées dans les 2 canaux $4(10,3-11,3 \mu \mathrm{m})$ et 5 $(11,5-12,5 \mu \mathrm{m}): \mathrm{Ts}=-2,2+3,6 \mathrm{~T}_{4}-2,6 \mathrm{~T}_{5}$ selon la méthode «split-window» (DESCHAMPS et PHULPIN, 1980) qui permet de corriger en partie les effets perturbateurs de l'atmosphère. Ces données sont ensuite retranscrites sur disquettes et analysées à la Station de Bioclimatologie I.N.R.A. d'Avignon sur le système Pericolor 1000 Numelec. Les corrections géométriques ont été affinées sur le Pericolor du CIRAD à Montpellier (procédure GEOREC) dans le but de rendre superposables toutes les scènes quelle que soit la date d'acquisition.
A partir des luminances mesurées dans le canal $\mathrm{Cl}$ (rouge 0,58 à $0,68 \mu \mathrm{m}$ ) et le canal C2 (proche infrarouge 0,72 à $1,10 \mu \mathrm{m}$ ), est ensuite effectué le calcul de l'indice de végétation IV correspondant à la différence normalisée :

$$
\mathrm{IV}=\frac{\mathrm{C} 2-\mathrm{Cl}}{\mathrm{C} 2+\mathrm{Cl}}
$$

\section{ANALYSE À L'ÉCHELLE DE LA PETITE RÉGION}

\section{A. Localisation géographique}

A l'intérieur de la zone d'étude globale, correspondant au Sud-Est de la France, nous nous sommes intéressés plus particulièrement à la basse vallée du Rhône, à relief faible à nul, et rassemblant des situations variées du point de vue de l'occupation du sol. Nous avons, en particulier, sélectionné une dizaine de zones plus réduites (de l'ordre de 50 à $200 \mathrm{~km}^{2}$ ), correspondant à des types d'occupation du sol fortement contrastés (fig. 1) :

- végétation herbacée spontanée fortement desséchée en été (Crau sèche) ;

- prairies permanentes et cultures maraîchères irriguées (Crau irriguée, Comtat, Nord des Alpilles) ;

- cultures céréalières (riz, blé, maïs) et vergers irrigués (Ouest Arles) ;

- vigne et arbres fruitiers en sec (Travaillan, Carpentras, Châteauneuf du Pape, Saint Chaptes).

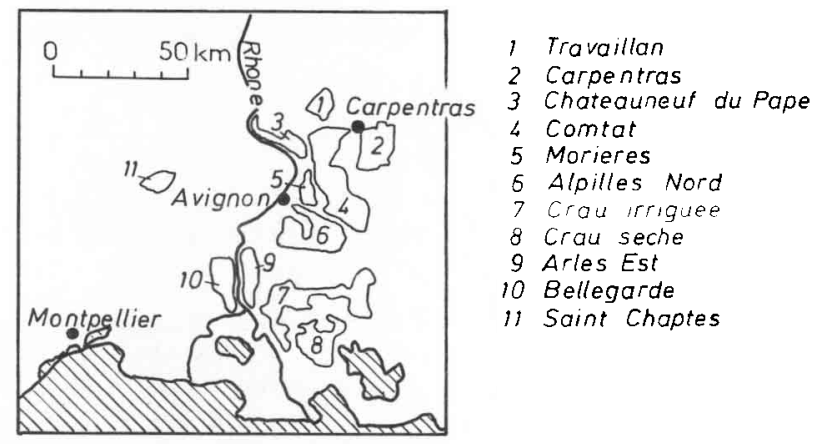

Figure 1

Implantation des zones étudiées dans le Sud-Est.

Location of studied zones in southeastern France.

Les figures 2 et 3 permettent d'apprécier l'évolution des indices de végétation de 5 de ces zones pour les données des étés 83 et 84 .

\section{B. Interprétation de l'évolution des indices}

L'échantillonnage temporel très irrégulier et différent d'un été sur l'autre ne permet pas une analyse complète, dans cette première phase. Il est possible, cependant, d'effectuer les observations suivantes :

- les classements relatifs d'indices traduisent nettement les différences de taux de couverture végétale : les zones de prairies (Crau irriguée-nord des Alpilles) sont caractérisées par des indices évoluant entre 0,30 et 0,40 , alors que la Crau sèche se situe aux alentours 


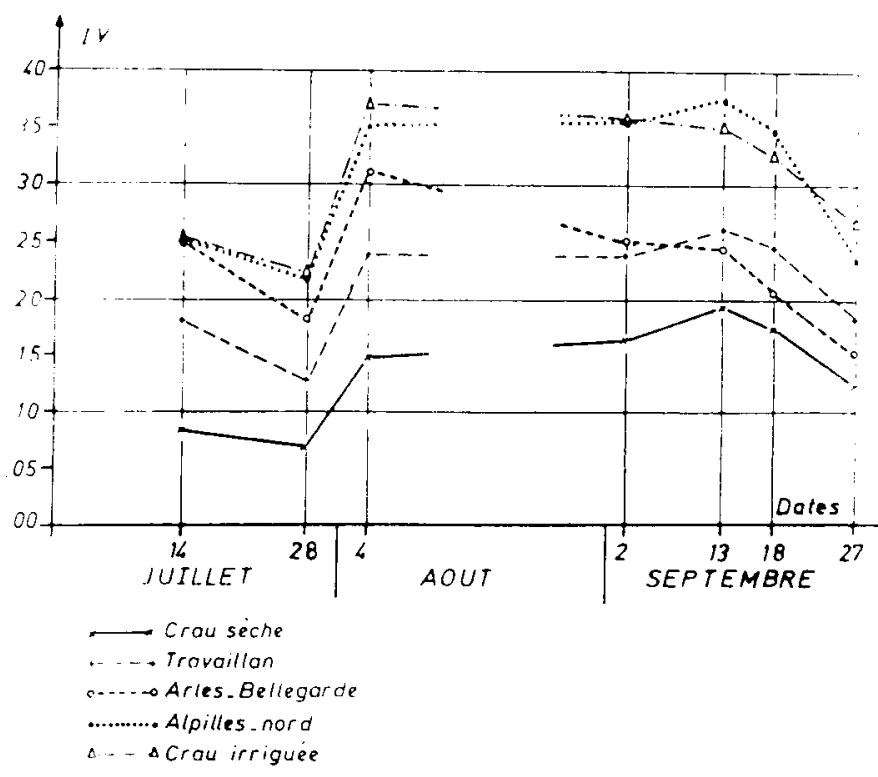

Figure 2

Evolution de l'indice normalisé IV au cours de l'été 83 pour 5 zones d'étude.

Change in the normalized difference index over summer 1983 for 5 test sites.

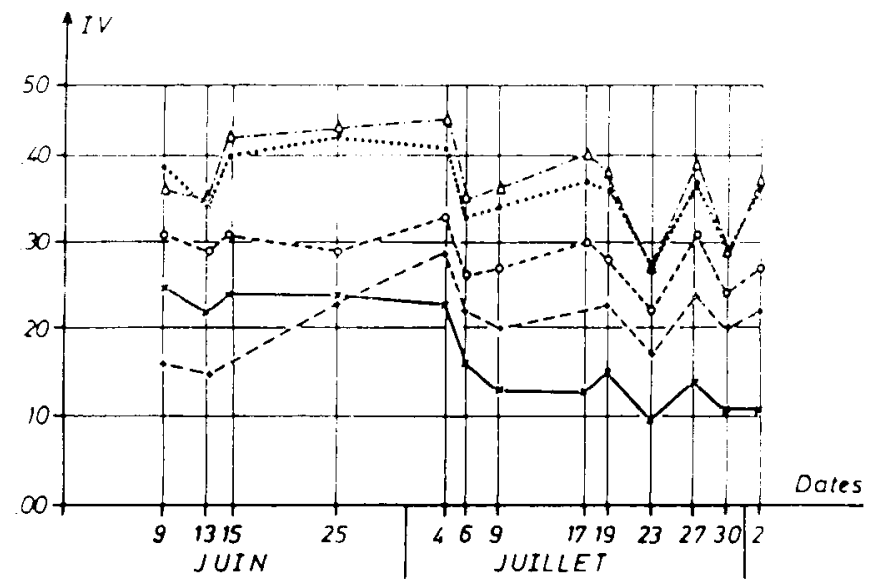

Crou seche

-... Travaillan

a...- Arles.Bellegarde

-.......... Alpilles nore

D.-.- Arau irriguae

Figure 3

Evolution de l'indice normalisé IV au cours de l'été 84 pour 5 zones d'étude.

Change in the normalized difference index over summer 1984 for 5 test sites.

de 0,10 en juillet. La zone de vignobles de Travaillan présente des indices faibles en juin (de l'ordre de 0,10 ) pour atteindre des niveaux de 0,20 et plus en juilletaoût. Quant à la zone d'Arles Bellegarde, elle montre un comportement intermédiaire : assez élevé en juinjuillet ( 0,3 et plus), l'indice est moyen en août et diminue nettement en septembre ;

- ces observations sont d'ailleurs confirmées par l'analyse des températures de surface obtenues à partir de l'IR thermique. Celles-ci ne sont pas discutées dans cet article, mais les résultats publiés par ailleurs (LAGOUARDE et al., 1985) montrent une liaison étroite avec les indices de végétation : aux faibles valeurs de celui-ci (Crau sèche par exemple) correspondent des valeurs élevées de $T_{S}$, traduisant une faible couverture végétale et un niveau d'évapotranspiration assez bas, alors que les fortes valeurs d'indice (Crau irriguée) correspondent à des $T_{S}$ plus faibles, liées à une forte évapotranspiration ;

- des «fluctuations » apparaissent dans l'évolution temporelle des indices. Dans la mesure où elles affectent l'ensemble des zones d'étude, et sur des périodes de temps très courtes (quelques jours), il est très vite apparu qu'elles devaient être attribuées à l'influence des facteurs atmosphériques affectant la transmission des luminances. Une analyse détaillée est présentée dans l'article cité ci-dessus (LAGOUARDE et al., 1985).

Nous nous contenterons ici, pour donner un aperçu de l'ordre de grandeur de cet artefact, de présenter la figure 4 , se référant à des zones boisées (forêts du Ventoux et garrigues de Nîmes) pour lesquelles on peut exclure l'hypothèse d'une variation effective du taux de couverture végétale sur de courtes séries. L'évolution simultanée du contenu en eau de l'atmosphère, traduite par l'épaisseur optique $\mathrm{w}$ en $\mathrm{cm}$, montre l'étroite correspondance avec les fluctuations d'indice : aux valeurs faibles de w (de l'ordre de $2 \mathrm{~cm}$ ) correspondent les pics d'indice, alors que les creux sont associés à des valeurs élevées de w $(3 \mathrm{~cm})$. Des méthodes de correction pourraient être développées, mais elles nécessitent la prise en compte systématique des radiosondages. Par ailleurs, les valeurs les plus basses sont affectées surtout par la présence de fortes concentrations en aérosols, conduisant à un trouble atmosphérique élevé, et pour lequel il n'apparaît pas de méthode simple de correction. Il est donc préférable d'éliminer les dates correspondantes.

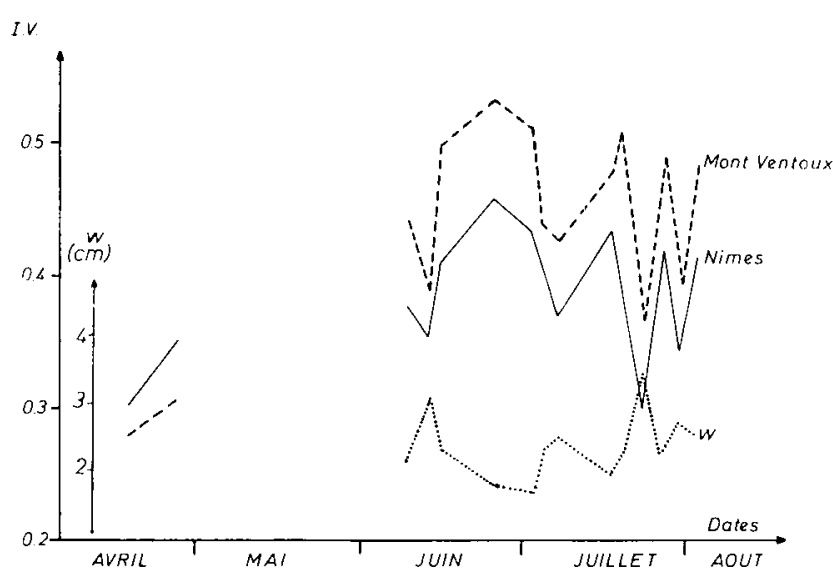

Figure 4

Evolution simultanée des indices normalisés des forêts du Ventoux et des garrigues de Nîmes et de l'épaisseur d'eau atmosphérique $w$ au cours de l'été 84.

Change in the indices for Ventoux (forest) and Nimes (scrub). Also shown is the precipitable water vapour for summer 1984.

L'existence de ces «fluctuations" doit donc être notée et gardée en mémoire pour l'analyse de l'évolution temporelle au cours de la saison de végétation, dans laquelle on ne s'attachera qu'aux évolutions de plus grande amplitude (de l'ordre de plus de 0,05). 
Au niveau de l'évolution temporelle sur la saison, certains faits peuvent être notés : dans le cas de la Crau sèche, un maximum marqué apparaît en juin 84 , en coïncidence avec les fortes pluies de mai et de début juin. Par contre, le dessèchement de juillet est notable pour les deux années, alors que les pluies du mois d'août permettent une légère remontée un peu plus tardive en 84 et non représentée sur la figure 3. L'été 83 fait apparaître une baisse progressive des indices au mois de septembre, qui traduit la fin de la saison de végétation.

\section{Intérêt et limites de cette analyse par zones}

Si cette analyse par zones permet de bien recouper les résultats publiés par la NOAA au niveau d'une surveillance globale de la végétation (en particulier, au moyen des produits standard disponibles sur bandes magnétiques et contenant les données d'indice au rythme hebdomadaire avec une résolution dégradée à environ $20 \mathrm{~km} \times 20 \mathrm{~km}$ ), il est permis de s'interroger sur l'utilité de telles données au niveau agronomique. Les caractéristiques de certaines petites régions en ce qui concerne le taux de couverture végétale (et l'état hydrique à partir de la température de surface) apparaissent nettement et peuvent constituer un support à un zonage agroécologique à une échelle de l'ordre de $1 / 500000^{\mathrm{e}}$, par exemple. Par contre, en ce qui concerne le suivi des cultures, l'information recueillie est trop grossière, compte tenu du mélange des cultures dans le contexte de l'occupation du territoire en France, pour cerner de près une réalité agricole. II nous a donc fallu pousser plus loin l'analyse, en essayant de délimiter des superficies plus réduites, correspondant à une occupation du sol par une culture, sinon totalement homogène, du moins largement majoritaire, pour évaluer réellement l'apport possible des données au niveau élémentaire de résolution de $1 \mathrm{~km}^{2}$.

\section{ANALYSE PLUS FINE À L'ÉCHELLE DES EXPLOITATIONS}

Cet objectif suppose l'identification préalable de " cibles" précises correspondant à une occupation du sol, sinon homogène, du moins par une culture très fortement dominante. Il a fait l'objet d'un travail de DEA (CLINET, 1985), qui a utilisé pour ce faire, les données de l'enquête "Utilisation du territoire " du Ministère de l'Agriculture, basée sur des sondages par photos aériennes. 32 petites surfaces, de 1 à $4 \mathrm{~km}^{2}$ (soit 1 à 4 pixels NOAA) ont ainsi été inventoriées. Des enquêtes complémentaires sur le terrain ont permis d'établir l'occupation effective au cours de l'année 1984.

L'ensemble des résultats obtenus est présenté dans le document précité. Nous présenterons ici seulement les résultats les plus significatifs, correspondant aux surfaces les plus " pures » pour l'occupation du sol. Leur localisation est indiquée sur la figure 5.

L'évolution des indices de végétation au cours de la saison 84 est reportée sur la figure 6. La comparaison des courbes des principales cultures étudiées permet de séparer nettement :

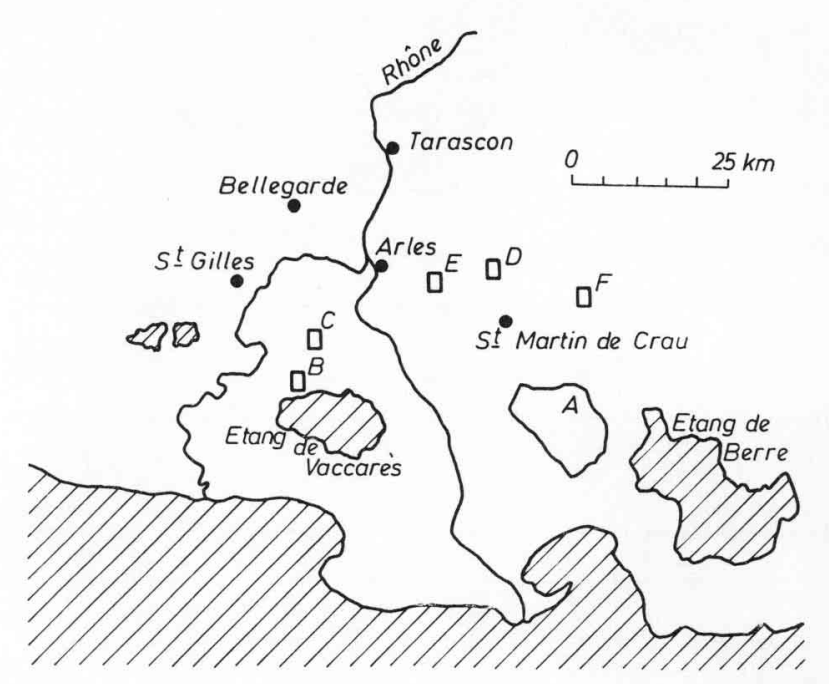

Figure 5

Localisation des surfaces élémentaires pour l'analyse à l'échelle fine.

A) Crau sèche ; B) blé 85 p. 100 Mas d'Agon; C) riz 80 p. 100 Rousty ; D) maïs 95 p. 100 Marais des Baux; E) prairies irriguées St-Martin; F) luzerne 80 p. 100 Mouriès.

Location of sites used in the small-scale analysis.

A) dry Crau ; B) wheat $85 \%$; C) rice $80 \%$; D) maize $95 \%$; E) irrigated grasslands; F) lucerne $80 \%$.

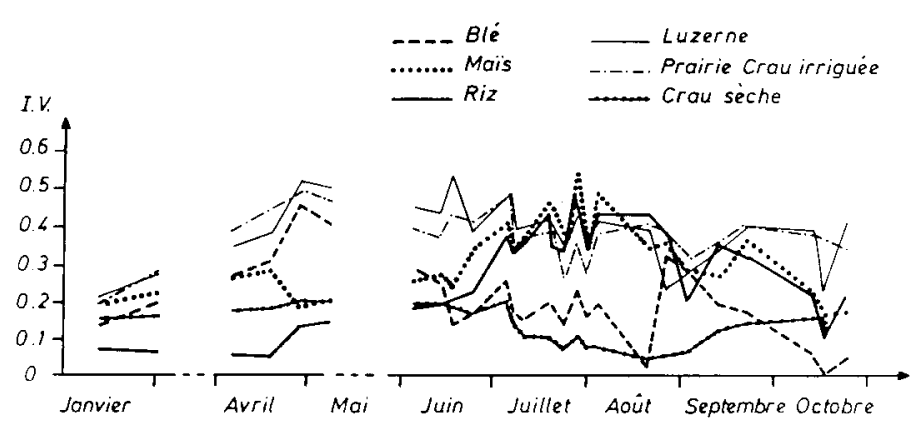

Figure 6

Evolution des indices de végétation des surfaces élémentaires au cours de l'année 1984.

(a) $A$ (Crau sèche) et $B$ (blé).

(b) $C$ (riz) et $D$ (mais).

(c) E (prairies irriguées) et $F$ (luzerne).

Pure crop vegetation indices over 1984.

(a) $A$ (dry Crau) and $B$ (wheat).

(b) $C$ (rice) and $D$ (maize).

(c) E (irrigated grasslands) and F (lucerne).

(a) : les surfaces à faible production de biomasse (Crau sèche), dont on peut noter le dessèchement en juillet et une légère remontée en septembre avec la reprise des pluies, ou à cycle précoce comme le blé, qui montre une élévation dès le mois d'avril et une chute courant juin, correspondant à la sénescence. Le mois de mai est malheureusement non représenté, aucune donnée n'étant disponible en raison de la couverture nuageuse. La reprise sur le blé, fin août, pourrait être due à 2 hypothèses : s'agit-il de la traduction de l'activité de la culture complémentaire (vigne) sur la zone ou de la repousse d'herbe à la suite des pluies ? L'effet est cependant trop marqué pour être un artefact ; 
(b) : les cultures plus tardives, comme le riz et le maïs, assez comparables, avec une phase de croissance de l'indice en juin, un plateau en juillet-août et une décroissance en septembre (la baisse d'indice observée sur maïs en avril-mai pouvant peut-être s'expliquer par une élimination des adventices à la suite des travaux du sol préalables au semis);

(c) : les fourrages, tels que luzerne ou prairies irriguées, également assez comparables et traduisant un indice élevé dès le mois d'avril, avec un léger creux fin août (il ne nous a pas été possible d'établir si les chutes d'indice à la mi-juin et mi-juillet correspondent effectivement à des dates de coupe).

\section{DISCUSSION ET CONCLUSIONS}

Ces résultats prouvent que, malgré la résolution spatiale limitée à $1 \mathrm{~km}^{2}$ et la variabilité du parcellaire français, les indices de végétation IV issus de NOAAAVHRR permettent de caractériser globalement l'évolution des couverts végétaux de surfaces relativement homogènes.

Il reste à établir quelles en sont les applications potentielles :

- on pourrait envisager de caractériser l'occupation du sol par le biais de ces profils temporels d'indice de végétation (traduisant le calendrier cultural). Cette perspective pourrait s'appliquer dans des pays à grande superficie agricole, peu cartographiés et pour obtenir des statistiques relativement grossières à faible coût. Par contre, au niveau du territoire français, cette application ne présente guère d'intérêt, par rapport aux possibilités de Landsat ou SPOT ;

- l'intérêt essentiel de NOAA résidant dans son suivi temporel, c'est à ce niveau que peut exister une utilisation opérationnelle. Il a été envisagé de diagnostiquer les avances ou retards des cultures. Cela semble possible, mais l'existence des "fluctuations » d'origine atmosphérique (dont on s'affranchit en partie en ne gardant que l'indice maximal sur des périodes de l'ordre de la semaine) rend difficile leur identification à moins de 10-15 jours, ce qui correspond à un événement climatique très marqué. Il semble plus envisageable, par contre, de pouvoir obtenir des informations sur l'extension spatiale de phénomènes tels que les sécheresses, de façon à diagnostiquer l'impact exact sur la biomasse produite. Cela suppose évidemment de disposer de cartes d'occupation de sol suffisamment précises et à jour ;

- c'est effectivement à ce niveau que se situe l'intérêt essentiel de ce suivi temporel des indices de végéta- tion. Si ceux-ci, à un instant donné, traduisent bien le degré de couverture du sol et l'importance de la biomasse présente, des travaux récents ont mis en évidence les relations qui existent avec le fonctionnement photosynthétique intégré sur quelques jours (voir le travail de modélisation de SelLERS, 1985) et la production de matière sèche (observations sur les pâturages néo-zélandais de TAYLOR et al., 1985) ; ils expliquent, en particulier, la décroissance de IV observée sur le blé, le maïs et le riz en début de sénescence et alors que la culture est à son indice foliaire maximal. Ainsi la valeur de l'indice intégré (ou moyenne) sur l'année peut être mise en relation avec la productivité globale (SEGUiN, 1985), avec une équivalence approximative $P(t / h a / a n)=(44,5 \pm 5) \overline{I V}$. L'indice intégré $\overline{I V}$ se situant, pour la Crau sèche, aux alentours de 0,10 et, pour les prairies irriguées, de 0,30 , cette équivalence, qui nécessite d'être reprise par d'autres travaux et est proposée à titre très exploratoire, correspondrait à des productions respectives de biomasse d'environ 5 et $15 \mathrm{t} / \mathrm{ha}$, ce qui est raisonnable, au moins au niveau des ordres de grandeur. Il est clair, cependant, qu'un tel indice intégré ne peut avoir qu'une valeur indicatrice.

Dans la mesure où ces hypothèses seraient précisées, l'objectif d'accéder au suivi et à la prévision de la production de biomasse serait réaliste. L'intérêt pour la plupart des productions françaises est limité, dans la mesure où la biomasse est rarement un facteur limitant. On peut penser bien sûr, à l'important secteur des pâturages, mais leur localisation dans des régions a priori relativement pluvieuses peut poser des problèmes de suivi à cause du fort ennuagement.

Par contre, la possibilité d'un tel suivi de la production de biomasse ouvre des perspectives importantes pour la prévision des rendements en agriculture moins intensive (le blé dans la plupart des pays producteurs, USA, URSS, Argentine, Australie, mais aussi une grande partie des productions des régions semi-arides). La mise au point de méthodes opérationnelles et de leur application par SPOT 3 (qui embarquera un instrument de suivi de la végétation, d'inspiration semblable à NOAA pour l'obtention régulière des indices de végétation) justifie la poursuite de ces travaux.

Reçu le 13 mai 1986.

Accepté le 10 septembre 1986.

\section{REMERCIEMENTS}

Ce travail a été effectué dans le cadre du projet sur l'utilisation des données satellitaires NOAA-AVHRR, coordonné par Y. KERR et G. SAINT au LERTS, avec l'appui financier de l'Action Thématique Programmée Télédétection Spatiale. 


\section{RÉFÉRENCES BIBLIOGRAPHIQUES}

Anglade I., 1985. Analyse de l'espace agricole par interprétation d'images satellite NOAA. Thèse $3^{\mathrm{e}}$ cycle. Université de ToulouseLe Mirail, 144 p. + 32 fig.

Clinet S., 1985. Suivi de l'évolution de types de couvert végétal par téléanalyse des données AVHRR du satellite NOAA-7. Mémoire de DEA en Géographie Physique. Université d'Aix-Marseille II, 118 p.

Deschamps P. Y., Phulpin T., 1980. Atmospheric correction of infrared measurement of sea surface temperature using channels at 3.7, 11 and $12 \mu$. Bound. Layer. Meteor., 18, 131-143.

Justice C. O., Townshend J. R. G., Holben B. N., Tucker C. T., 1985. Analysis of the phenology of global vegetation using meteorological satellite data. Int. Journ. Rem. Sens., 6 (8), 1271-1318.

Lagouarde J. P., Seguin B., Kerr Y., Gandia S., Roy S., 1985. Analyse des images NOAA dans le Sud-Est de la France. In : Télédétection et gestion des ressources : l'aspect opérationnel, Vol. 5, Bernierd, Lessard et Gagnon ed. C.R. du congrès de Chicoutimi organisé par l'A.Q.T., mai 1985, 147-157.

Saint G., Podaire A., Fournier Ph., Meyer-Roux J., Cordier P., 1984. Comparaison des capteurs SPOT-HRV et Landsat 4-TM pour l'obtention de statistiques agricoles. $2^{e}$ Coll. Int. Signatures Spectra- les d'Objets en Télédétection. Bordeaux, sept. 1983, C. R. ed. par I.N.R.A., 331-341.

Seguin B., 1983. Apports possibles de la télédétection en agroclimatologie. Séminaire Comm. Agrométéo. I.N.R.A., Paris, sept. 1983. C. $R$. ed. par I.N.R.A. Applications de la télédétection à l'agriculture, 151-155.

Seguin B., 1986. On the relationship between time integrated NDVI (Normalized Difference Vegetation Index) and global productivity. Proc. ISLSCP Conf. Rome, Italy, 2-6 Dec. 1985. ESA-SP 248 , p. 299.

Sellers P. J., 1985. Canopy reflectance, photosynthesis and transpiration. Int. Journ. Rem. Sens., 6 (8), 1335-1373.

Taylor B. F., Dini P. W., Kidson J. W., 1985. Determination of seasonal and interannual variation in New Zealand pasture growth from NOAA-7 data. Rem. Sens. Env., 18, 177-192.

Vidal A., Kerr Y., Lagouarde J. P., Seguin B., 1986. Télédétection et bilan hydrique : utilisation combinée d'un modèle agrométéorologique et des données de l'IR thermique du satellite NOAAAVHRR. Agric. Forest. Meteor. sous presse. 